\title{
THE N2K CONSORTIUM. VI. DOPPLER SHIFTS WITHOUT TEMPLATES AND THREE NEW SHORT-PERIOD PLANETS ${ }^{1}$
}

\author{
John Asher Johnson, ${ }^{2}$ Geoffrey W. Marcy, ${ }^{2}$ Debra A. Fischer, ${ }^{3}$ Gregory Laughlin, ${ }^{4}$ R. Paul Butler, ${ }^{5}$ \\ Gregory W. Henry, ${ }^{6}$ Jeff A. Valenti, ${ }^{7}$ Eric B. Ford, ${ }^{2}$ Steven S. Vogt, ${ }^{4}$ and Jason T. Wright ${ }^{2}$ \\ Received 2006 February 21; accepted 2006 April 14
}

\begin{abstract}
We present a modification to the iodine cell Doppler technique that eliminates the need for an observed stellar template spectrum. For a given target star, we iterate toward a synthetic template spectrum beginning with an existing template of a similar star. We then perturb the shape of this first-guess template to match the program observation of the target star taken through an iodine cell. The elimination of a separate template observation saves valuable telescope time, a feature that is ideally suited for the quick-look strategy employed by the "Next 2000 Stars" (N2K) planet search program. Tests using Keck HIRES (High Resolution Echelle Spectrometer) spectra indicate that synthetic templates yield a short-term precision of $3 \mathrm{~m} \mathrm{~s}^{-1}$ and a long-term, run-to-run precision of $5 \mathrm{~m} \mathrm{~s}^{-1}$. We used this new Doppler technique to discover three new planets: a $1.50 M_{\mathrm{J}}$ planet in a 2.1375 day orbit around HD 86081; a $0.71 M_{\mathrm{J}}$ planet in circular, 26.73 day orbit around HD 224693; and a Saturn-mass planet in an 18.179 day orbit around HD 33283. The remarkably short period of HD $86081 \mathrm{~b}$ bridges the gap between the extremely short period planets detected in the Optical Gravitational Lensing Experiment (OGLE) survey and the 16 Doppler-detected hot Jupiters $(P<15$ days), which have an orbital period distribution that piles up at about 3 days. We have acquired photometric observations of two of the planetary host stars with the automated photometric telescopes at Fairborn Observatory. HD 86081 and HD 224693 both lack detectable brightness variability on their radial velocity periods, supporting planetary-reflex motion as the cause of the radial velocity variability. HD 86081 shows no evidence of planetary transits in spite of a $17.6 \%$ transit probability. We have too few photometric observations to detect or rule out transits for HD 224693.
\end{abstract}

Subject headings: planetary systems: formation — stars: individual (HD 33283, HD 86081, HD 224693) — techniques: radial velocities

Online material: machine-readable table

\section{INTRODUCTION}

Of the 180 planets that have been found orbiting stars within $200 \mathrm{pc}$ of the Sun, ${ }^{8}$ the vast majority have been discovered by measuring Doppler reflex motion of their parent stars (Butler et al. 2006). Effectively detecting the relatively small Doppler shifts caused by planet-sized bodies requires velocity precision of order $1 \mathrm{~m} \mathrm{~s}^{-1}$. This corresponds to measuring shifts in spectral lines to within $10^{-3}$ of a resolution element for modern echelle spectrometers such as Keck HIRES (Vogt et al. 1994). Such a feat requires intimate knowledge of the wavelength scale of the spectrometer detector. However, the wavelength scale is not constant with time. The instrument is subject to changes on timescales as short as hours, or even minutes, due to effects such as

\footnotetext{
${ }^{1}$ Based on observations obtained at the W. M. Keck Observatory, which is operated jointly by the University of California and the California Institute of Technology.

2 Department of Astronomy, University of California, Mail Code 3411, Berkeley, CA 94720.

3 Department of Physics and Astronomy, San Francisco State University, San Francisco, CA 94132.

${ }^{4}$ UCO/Lick Observatory, University of California at Santa Cruz, Santa Cruz, CA 95064.

5 Department of Terrestrial Magnetism, Carnegie Institution of Washington, 5241 Broad Branch Road NW, Washington, DC 20015-1305.

${ }^{6}$ Center of Excellence in Information Systems, Tennessee State University, 3500 John A. Merritt Boulevard, Box 9501, Nashville, TN 37209.

7 Space Telescope Science Institute, 3700 San Martin Drive, Baltimore, MD 21218.

${ }^{8}$ References to published papers and updates on orbital parameters can be found at http://exoplanets.org.
}

telescope flexure and thermal expansion. It is therefore necessary to measure the wavelength scale for each observation using a reference spectrum obtained simultaneously with the stellar spectrum.

One of the most successful and widely used precision velocity methods is the iodine cell technique (Butler et al. 1996, hereafter B96). The B96 technique uses an iodine cell placed in front of the entrance slit of an echelle spectrometer, which imprints a dense set of narrow molecular lines on each stellar spectrum. Velocity information is extracted by fitting a model consisting of a stellar template spectrum multiplied by an iodine reference spectrum and convolved with an instrumental profile, or pointspread function (PSF). The shape of the PSF, wavelength scale of the spectrometer, and Doppler shift of the stellar template are left as free parameters in the fit.

The stellar template spectrum is an observation of the target star obtained without the iodine cell, with the instrumental profile deconvolved. Because numerical deconvolution algorithms are highly susceptible to noise amplification (e.g., Starck et al. 2002), template observations must have a high signal-to-noise ratio ( $\mathrm{S} / \mathrm{N} \gtrsim 500)$ and high spectral resolution $(\lambda / \Delta \lambda \gtrsim 80,000)$. Both of these requirements result in longer exposure times compared to program observations made through the iodine cell.

One observing program for which this telescope time overhead is prohibitive is the "Next 2000 Stars" (N2K) planet search (Fischer et al. 2005, hereafter Paper I). N2K is an international consortium designed to search for short-period planets $(P<15$ days, commonly known as "hot Jupiters") orbiting metal-rich stars residing within $110 \mathrm{pc}$ of the Sun. As telescope time is allocated at each of three major telescopes-Keck, Magellan, and Subaru—each 
target star is monitored for three consecutive nights. Those stars exhibiting radial velocity (RV) variations between 20 and $250 \mathrm{~m} \mathrm{~s}^{-1}$ are flagged for follow-up spectroscopic observations to search for a full orbital solution. Planet candidates also receive photometric monitoring with the automatic photoelectric telescopes (APTs) at Fairborn Observatory (Henry 1999) to search for transit events. To date, this "quick look" observing strategy has resulted in the detection of four short-period planets (Paper I; Fischer et al. 2006), one of which, HD 149026, transits its host star (Sato et al. 2005b).

Most N2K target stars receive only three RV observations, which means that template observations represent more than $25 \%$ of the telescope time required for the program. Since template observations contain no inherent velocity information, it would be highly advantageous to bypass them by measuring Doppler shifts with respect to synthetic reference spectra. Using this motivation, we have developed a modification to the B96 velocity method whereby we derive the shape of the intrinsic stellar spectrum directly from normal iodine cell observations. This synthetic, or "morphed," template is then used in place of a deconvolved template in the B96 modeling process.

We present an overview of the forward modeling procedure used to produce morphed templates in $\S 2$. In $\S 3$ we show the results of several tests of both the short- and long-term precision attainable from morphed templates using Keck HIRES observations of a set of velocity standard stars. In $\S 4$ we detail how this new method is currently being used in the N2K planet search. We also report the detection of three new short-period planets, and describe the results of our photometric monitoring of the host stars for transit events. Finally, in $\S 5$ we summarize our results, propose other potential applications for spectral morphing, and discuss the new planet discoveries with respect to the growing statistical ensemble of short-period exoplanets.

\section{SPECTRAL MORPHING}

The B96 precision velocity technique employs a temperaturecontrolled Pyrex cell containing gaseous molecular iodine placed at the entrance slit of a spectrometer. The iodine lines imprinted on the stellar spectrum serve as a robust wavelength fiducial against which the Doppler shift of the stellar lines is measured. The Doppler information from each iodine observation is extracted using a forward modeling algorithm described in detail by B96 and summarized below.

Depending on the format of the echelle spectrometer, the stellar spectra are broken into 600-1000 $2 \AA$ wide spectral chunks, and each chunk is modeled as

$$
I_{\mathrm{obs}}(\lambda)=k\left[T_{I 2}(\lambda) \operatorname{DST}(\lambda+\Delta \lambda)\right] * \mathrm{PSF}
$$

where $k$ is a normalization factor, PSF is the model of the spectrometer's instrumental response, and the asterisk denotes a convolution. The fitting procedure is illustrated in Figure 1. The Doppler shift, $\Delta \lambda$, is applied to the deconvolved stellar template (DST) while the iodine reference spectrum, $T_{I 2}(\lambda)$, determines the wavelength scale of the spectral chunk. The stellar template is multiplied by the iodine template, and the product is convolved with the PSF and fit to the observed spectrum.

The fitting procedure requires two template spectra: the deconvolved stellar template and the iodine transmission spectrum. The iodine spectrum has been measured at high resolution $(R>200,000)$ and high signal-to-noise $(\mathrm{S} / \mathrm{N}>700)$ using the Fourier Transform Spectrometer at Kitt Peak National Observatory. While the same iodine template can be used for every RV observation, the stellar template must be measured for each in-

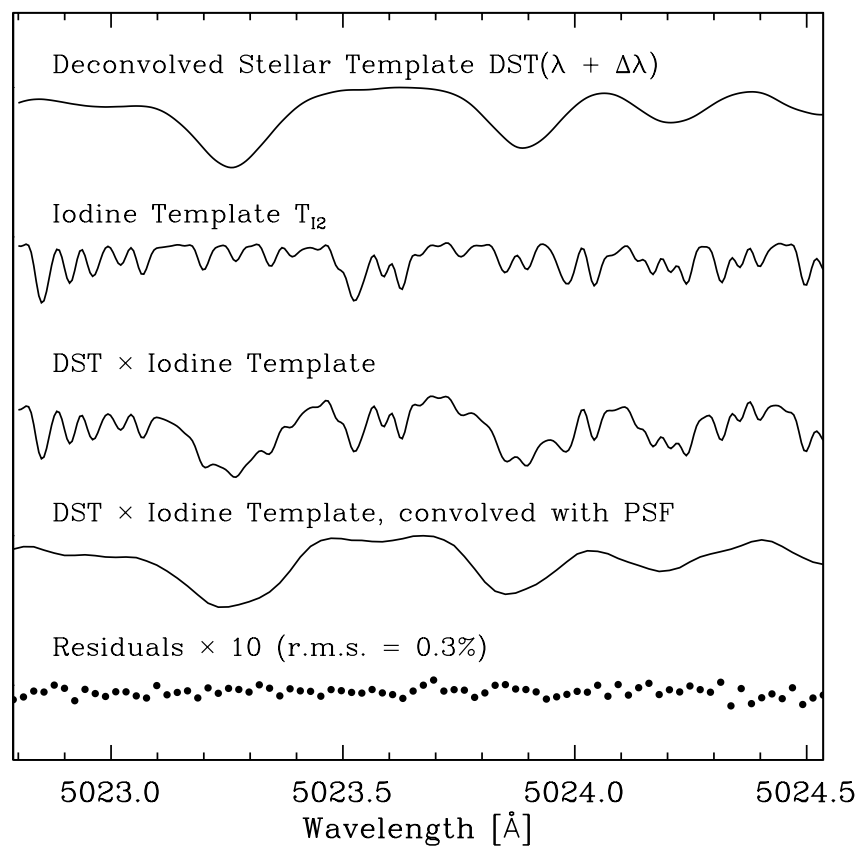

FIG. 1.-Illustration of the iodine cell Doppler model. From top to bottom, the shifted deconvolved stellar template is multiplied by the iodine reference spectrum. The product is then convolved with the spectrometer PSF and fit to the stellar spectral observation obtained through the iodine cell. The residuals from the fit are shown at the bottom.

dividual target star. Stellar template observations are made without the iodine cell in the spectrometer light path, and are obtained with higher resolution and signal-to-noise ratios than typical iodine observations. Templates also require bracketing iodine spectra of rapidly rotating B stars, which are used to measure the spectrometer PSF and wavelength scale at the approximate time of the template observation. The PSF is then removed using a modified Jansson deconvolution algorithm (Jansson 1995).

Our modification to the B96 reduction procedure is to derive the DST directly from an iodine observation, thereby circumventing the separate template observation for each target (see Sato et al. [2002] for a similar method). We use a modeling procedure similar to equation (1), replacing the DST with a synthetic, or "morphed," template spectrum. The shape of the spectrum is varied until, when multiplied by the iodine spectrum and convolved with the PSF, a fit is obtained to the stellar iodine observation. This morphed deconvolved template is then substituted for the DST in equation (1) during the velocity analysis of the target star's iodine observations.

The fitting procedure requires an accurate initial guess of the shape of the morphed DST. A spectrum derived from a model stellar atmosphere would seem like an ideal choice, since each individual spectral line could be described analytically. But the fit of equation (1) to the observed spectrum is highly sensitive to the exact positions of spectral lines in the DST. Small mismatches between model and observed spectra caused by effects such as convective blueshift can be compensated by asymmetric adjustments to the PSF model, which can in turn lead to spurious Doppler shifts (e.g., Winn et al. 2005). Modern stellar atmosphere models cannot accurately account for the identification and exact wavelengths of each of the thousands of lines spanning the $1000 \AA$ wide iodine region.

Instead, we find that an existing observed DST with characteristics similar to the target star works the best as the initial guess of the shape of morphed template. Stars with similar surface gravity, effective temperature and chemical composition differ 
only slightly in the depth and width of individual spectral lines. We have built a library of DSTs for 42 stars spanning the main sequence and subgiant branch of the H-R diagram, with Hipparcos colors $0.55<B-V<1.3$ (ESA 1997), and projected equatorial rotation velocities $V_{\text {rot }} \sin i<2 \mathrm{~km} \mathrm{~s}^{-1}$ as measured by Valenti $\&$ Fischer (2005). The library templates were observed as part of the regular California and Carnegie Planet Search (CCPS; Vogt et al. 2000; Marcy et al. 2005b), using the Keck HIRES echelle spectrometer with the 0."38 wide E2 decker, which yields a spectral resolution of $\lambda / \Delta \lambda \sim 110,000$ at $5500 \AA$ A. Each library template observation consists of three to five sequential exposures, with co-added signal-to-noise ratios ranging from 800 to 1000 . The PSF is derived from a set of bracketing B star/iodine spectra and removed from the template spectrum using the modified Jansson deconvolution algorithm (B96).

The morphing procedure begins by selecting three to six library template stars with Hipparcos colors and absolute visual magnitudes similar to the target star. The non-deconvolved template spectra of the candidate library stars are then cross-correlated against the target star's "pseudotemplate," which is created by dividing the star-plus-iodine observation by a B star/iodine spectrum from the same observing run. The library template that yields strongest cross-correlation peak, averaged over all orders, is chosen as the initial guess for the shape of the deconvolved stellar template.

With the first-guess spectrum established, the model we use to derive the morphed DST is

$$
I_{\mathrm{obs}}(\lambda)=k\left[T_{I 2}(\lambda) M(\mathrm{DST})\right] * \mathrm{PSF},
$$

where $M(\mathrm{DST})$ represents a transformation of the library deconvolved template. We do not allow the PSF to vary, but instead derive its shape from a B star/iodine spectrum obtained near the time of the observation of the target star. As in equation (1), the DST is shifted by $\Delta \lambda$. But in equation (2), the shape of the spectrum is also allowed to vary by adjusting the depths of individual lines and globally broadening the spectrum with a rotational broadening kernel corresponding to an equatorial rotation velocity $V_{\text {rot }}$.

As with the B96 method, the fit is carried out on individual $2 \AA$ wide spectral chunks. However, in order to modify the line depths of the DST, the spectral chunks are further divided into individual line segments using an automated search algorithm. The algorithm first locates the minima in the DST that lie further than $4 \sigma$ below the nominal continuum level, and then traces along the line wings until either the continuum or a neighboring line segment is encountered. Neighboring line segments corresponding to line blends are merged and the blend is treated as a single line, a compromise necessary to avoid cross talk between the two segments.

The transformation function can be expressed as

$$
M(\mathrm{DST})=R\left(V_{\mathrm{rot}}\right) * \sum \exp \left\{-f_{i} \ln \left[\mathrm{DST}_{i}(\lambda+\Delta \lambda)\right]\right\},
$$

where $\mathrm{DST}_{i}$ is the $i$ th spectral segment of the library DST, which is scaled by a factor $f_{i}$. The rotational broadening kernel, $R\left(V_{\text {rot }}\right)$, is computed with a linear limb-darkening coefficient appropriate for the star's $B-V$ color and the mean wavelength of the iodine spectral region (Gray 1992). Figure 2 shows an example of a morphed deconvolved template, together with the resulting fit of equation (2) to an observed stellar iodine observation and the fit residuals.

Since the library DST is continuum normalized, the logarithmic term in equation (3) can be thought of as a pseudo-optical depth under optically thin conditions. Our choice of this functional form

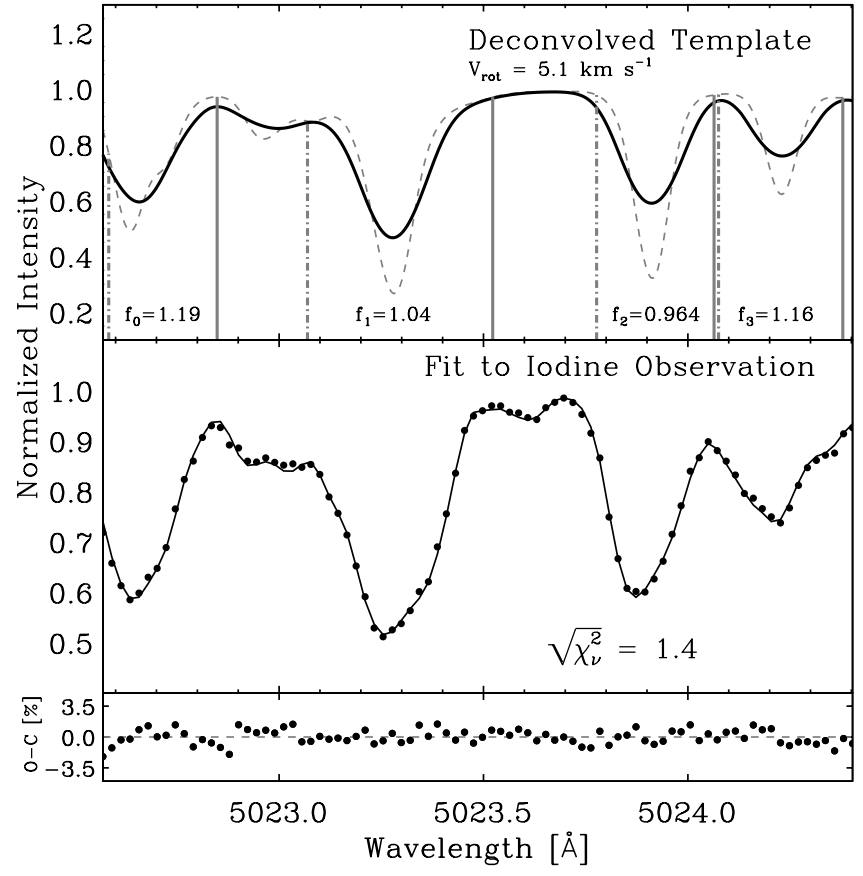

FIG. 2.-Illustration of spectral morphing algorithm. Top: The first-guess deconvolved stellar template (DST; dashed lines) compared to the modified synthetic DST (solid line). The synthetic spectrum has been rotationally broadened by $5.1 \mathrm{~km} \mathrm{~s}^{-1}$ and the four spectral segments that have been identified as individual lines have been adjusted by a pseudo-optical depth factor $f_{i}$. The borders of the four line segments are marked on the left (dot-dashed line) and right (solid line). Middle: The DST multiplied by the iodine reference spectrum (solid line), convolved by the PSF model, and shifted to fit the stellar observation (dots). Bottom: The fit residuals.

is obviously not motivated by physical considerations since the spectral lines in the template are not optically thin. Instead, we chose it because it has the desirable behavior of leaving the continuum unchanged while the depth adjustment is applied smoothly from the line wings to the core. This has the effect of stretching the line in a somewhat elastic fashion. To enforce continuity from one line segment to the next, each segment has a pseudocontinuum level such that portions of $\mathrm{DST}_{i}$ that lie above this level are left unchanged. For a given line segment, the pseudocontinuum is the lesser of either the value at the left segment boundary or $98 \%$ of the stellar continuum.

In most cases, particularly with N2K targets, we derive the morphed DST from a single iodine observation. However, since the fitting procedure is carried out in the rest frame of the library star, a refined solution can be obtained by fitting equation (2) to multiple spectral observations. The composite morphed DST is formed by replacing the line depths, $f_{i}$, in equation (3) with the weighted average,

$$
\overline{f_{i}}=\frac{\sum f_{i, j} / \sigma_{j}^{2}}{\sum 1 / \sigma_{j}^{2}},
$$

where $f_{i, j}$ is the line depth adjustment for the $i$ th segment of the fit to the $j$ th observation, and $\sigma_{j}$ is the standard deviation of the residuals of the fit of equation (2). The broadening kernel is similarly averaged over all observations.

\section{TESTS}

The N2K planet search uses a quick-look observing strategy to efficiently search for short-period planet candidates within a set of $\sim 2000$ target stars. Stars are typically monitored over 


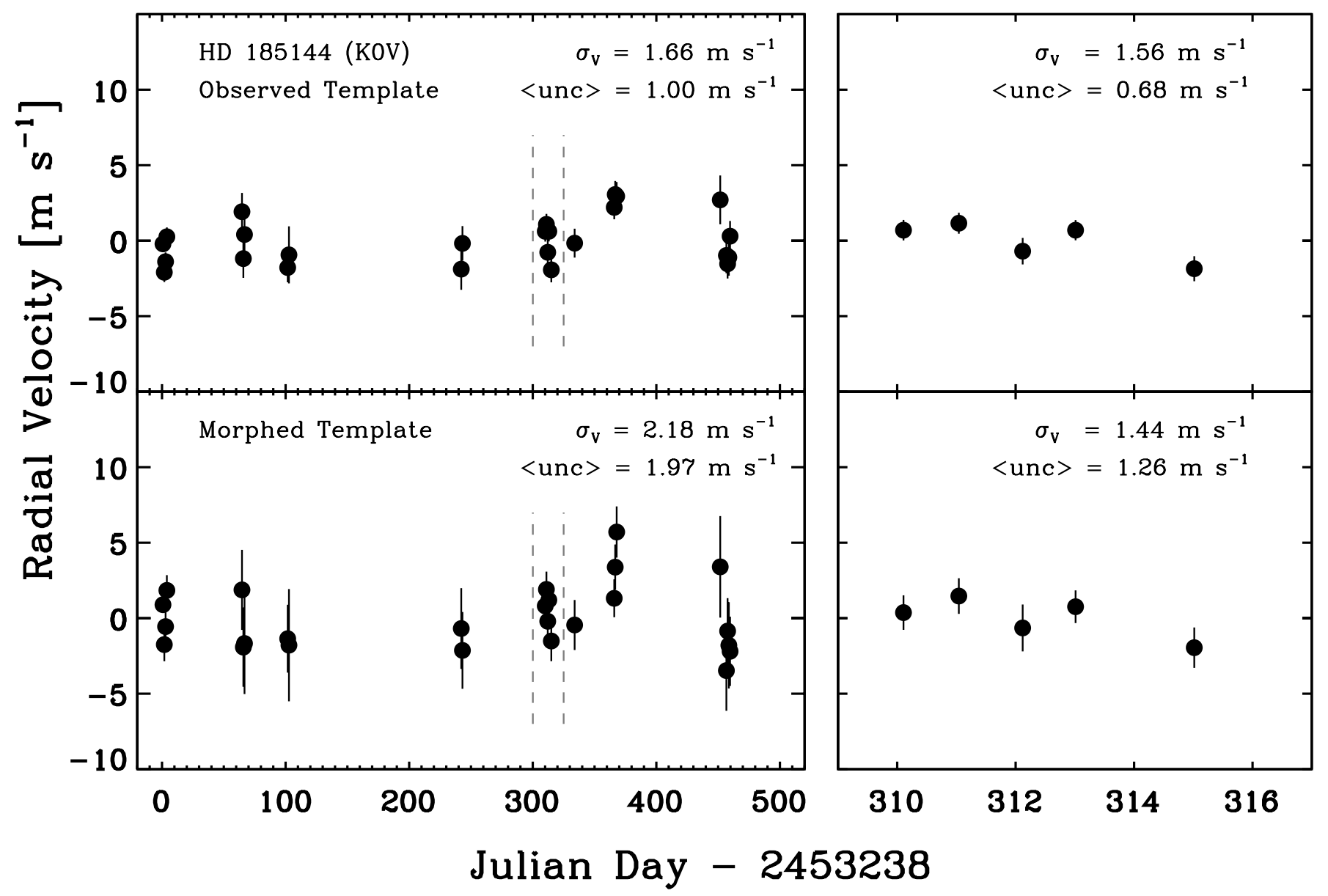

FIG. 3.-Comparison of velocities derived from a standard observed template (top) and a synthetic morphed template (bottom) for HD 185144 (spectral type K0 V). The left panels show the longterm velocity precision for each type of template and the right panels show the precision during a single observing run (corresponds to area between vertical dashed lines in left panel); 〈unc〉 is the mean internal uncertainty.

three consecutive nights, and those exhibiting conspicuous RV variations (typically $>5 \sigma$ ) are flagged for follow-up observations. A Jupiter-mass planet in a short-period ( $<15$ day) orbit will produce velocity variations of order hundreds of meters per second, necessitating only modest velocity precision. However, the number of extrasolar planets rises sharply with decreasing planet mass as $d N / d M \propto M^{-1.0}$ (Marcy et al. 2005a), which makes it crucial that we attain the highest possible precision in order to detect Saturn-mass planets. For example, a $0.3 M_{\mathrm{J}}$ planet in a 15 day orbit around a solar-mass star induces RV variations with an amplitude of only $25 \mathrm{~m} \mathrm{~s}^{-1}$. Reliably detecting this relatively low amplitude signal from three velocity measurements made during consecutive nights requires a short-term measurement precision better than $\sim 5 \mathrm{~m} \mathrm{~s}^{-1}$.

We are also interested in the long-term performance of morphed templates. While most stars are monitored over consecutive nights, the velocity measurements of some stars have to be split up over several runs when telescope time is lost to weather. Also, it would be ideal if morphed DSTs are stable enough from one run to the next to allow for follow-up without having to obtain a traditional template observation. In the following subsections we compare both the short- and long-term velocity performance of morphed DSTs to observed templates.

\subsection{Standard Stars}

In 2004 August, the Keck HIRES spectrometer was upgraded with a new detector. The previous $2 \mathrm{~K} \times 2 \mathrm{~K}$ pixel Tektronix $\mathrm{CCD}$ was replaced by an array of three $4 \mathrm{~K} \times 2 \mathrm{~K}$ pixel MIT-LL CCDs. The new detector produces much higher velocity precision due to its improved charge transfer efficiency and charge diffusion characteristics, smaller resolution elements (15 $\mu \mathrm{m}$ pixels compared to the old $24 \mu \mathrm{m}$ pixels), higher quantum efficiency, increased spectral coverage, and lower read noise.

Since the detector upgrade, we have monitored a set of 32 chromospherically inactive stars as part of the CCPS in order to evaluate the RV precision of our Doppler reduction pipeline. Each of these RV "standard" stars has received intensive monitoring during at least one observing run, with multiple observations spanning consecutive nights. In addition, 14 of the stars have also been observed during at least five separate observing runs spanning more than a year and a half. Typical signal-to-noise ratios range from 600 to more than 1000, depending on the star's apparent magnitude, with a spectral resolution $\lambda / \Delta \lambda=80,000$ at $5500 \AA$.

\subsection{Velocity Comparisons}

We used these "standard" stars to perform a differential evaluation of the velocity precision of morphed versus observed DSTs. Figure 3 shows an RV plot for one of the more intensely observed standard stars, HD 185144 ( $\sigma$ Dra; spectral type K0 V). The top panels show the velocities measured using an observed DST and the bottom panels show the velocities derived from a morphed DST. The velocities measured from a morphed template exhibit a long-term rms scatter of $2.16 \mathrm{~m} \mathrm{~s}^{-1}$, compared to $1.66 \mathrm{~m} \mathrm{~s}^{-1}$ 


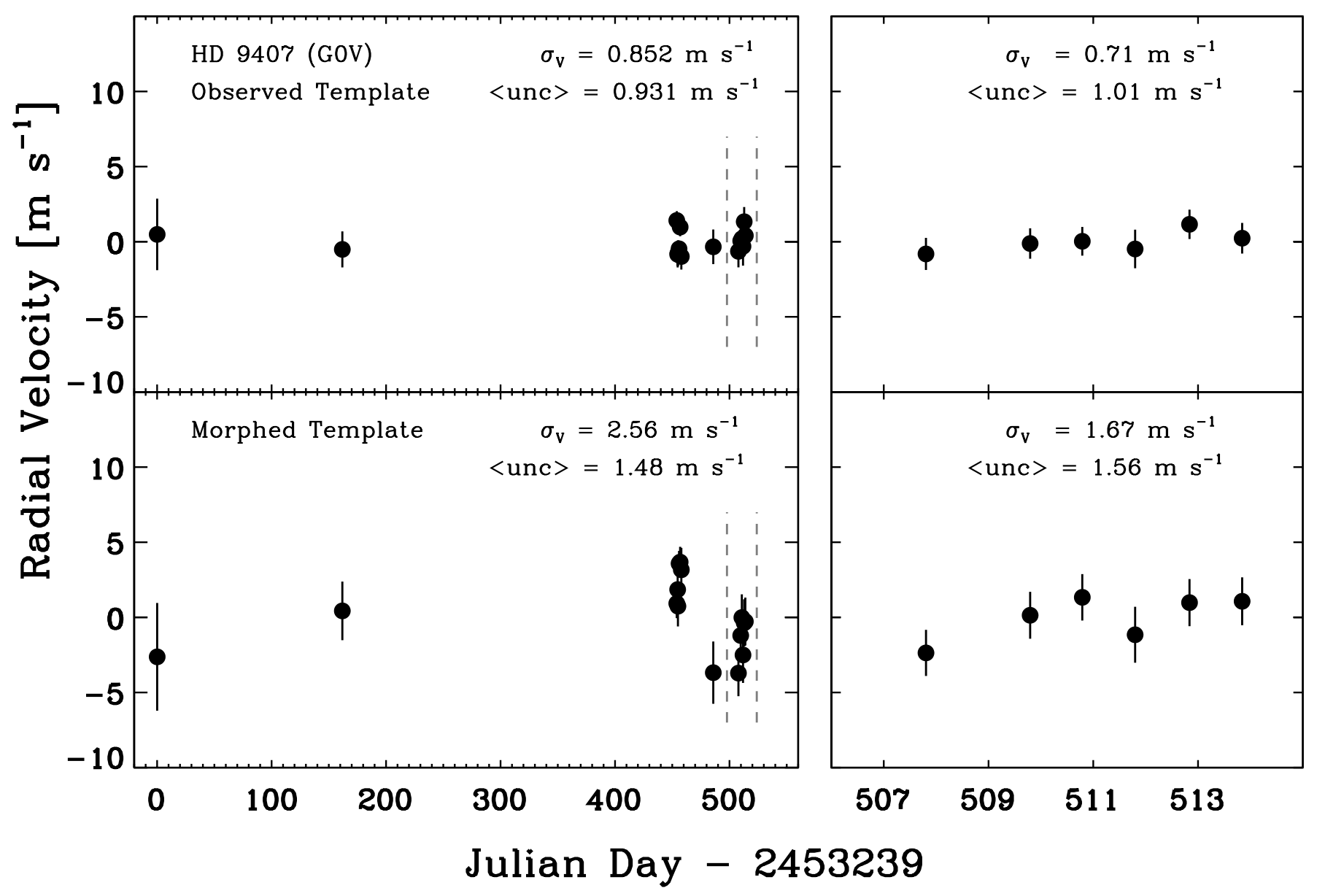

FIG. 4.-Same as Fig. 3, but for HD 9407 (spectral type G0 V).

for the observed template. The short-term rms (right panels) for the morphed template velocities is nearly identical to that of the observed template $\left(1.44 \mathrm{~m} \mathrm{~s}^{-1}\right.$ vs. $\left.1.56 \mathrm{~m} \mathrm{~s}^{-1}\right)$. Figure 4 shows a similar velocity comparison for HD 9407 (G0 V). In this case, the observed-template velocities have rms $<1 \mathrm{~m} \mathrm{~s}^{-1}$ for both the short- and long-term test cases, while the scatters in the morphedtemplate velocities are about 2 and 3 times larger, respectively.

Not surprisingly, the mean internal uncertainty, $\langle$ unc $\rangle$, of the morphed velocities is larger than the internal errors produced by the observed templates. This is likely the result of small differences between the morphed DST and the true stellar spectrum, which are not adequately accounted for by equation (2). These mismatches are also likely responsible for the systematic offsets from run to run in the HD 9407 velocity measurements shown in Figure 4 between days 450 and 550.

Figure 5 shows histograms of the short-term (single run) velocity scatter, $\sigma_{V}$, measured for the full set of 32 standard stars using morphed and observed templates. Figure 6 shows a similar comparison for the subset of 14 stars with $\sim 1.5$ years of observations spanning more than five separate observing runs. A comparison of the mean velocity scatter shows that morphed templates perform within a factor of 2 of observed templates, with a typical precision of $\sim 3 \mathrm{~m} \mathrm{~s}^{-1}$ within a single observing run and a longterm precision of $\sim 5 \mathrm{~m} \mathrm{~s}^{-1}$.

It should be cautioned that Figures 5 and 6 do not represent unbiased tests of the absolute precision attainable with the B96 technique. Such a test is complicated by differences in the quality of observations from one run to the next, the time coverage and frequency of the observations, and the possible presence of undetected low-mass planets. Instead, our tests represent a differential comparison of the precision produced by morphed and observed templates. From this test we can conclude that morphed templates are well suited to the needs of the N2K observing program, providing the necessary short-term precision for the initial

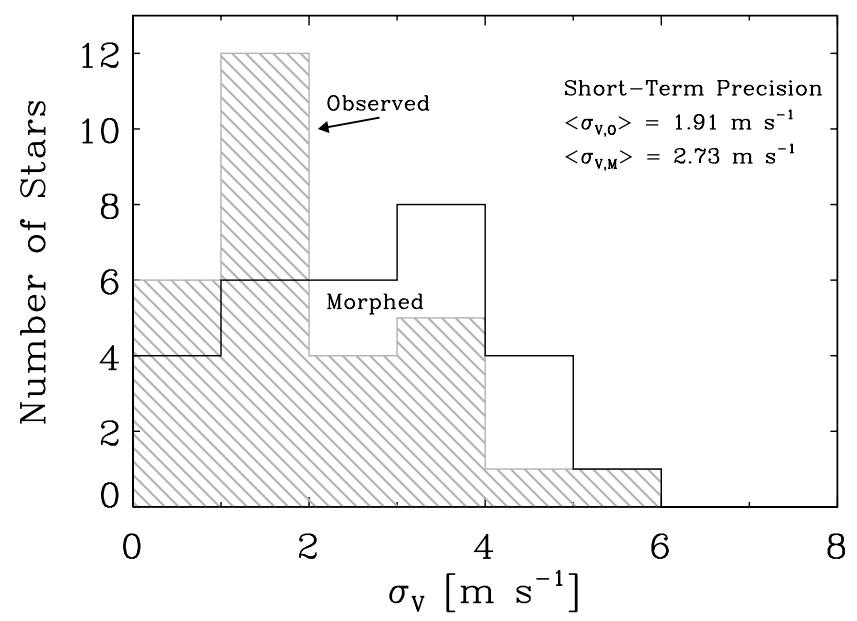

FIG. 5.-Histograms comparing the shortterm (5 night) velocity precision of morphed vs. observed (shaded) templates for a set of 32 chromospherically quiet radial velocity standards. The mean rms scatter of the velocities derived from morphed and observed templates is given by $\left\langle\sigma_{V, M}\right\rangle$ and $\left\langle\sigma_{V, O}\right\rangle$, respectively. 


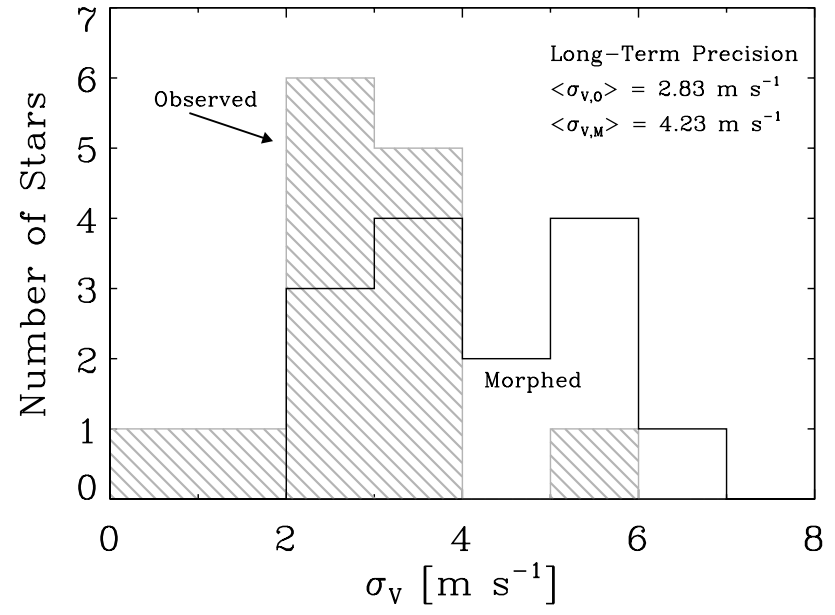

FIG. 6.-Same as Fig. 5, but for a subset of 14 standard stars observed during more than five observing runs spanning $\sim 1.5$ years.

detection of Saturn-mass planets, and long-term precision adequate for monitoring planet candidates from one run to the next.

\section{APPLICATION TO N2K}

During the 2005B Keck observing semester, we observed 63 metal-rich stars chosen based on the criteria described in Paper I (see also Robinson et al. 2006; Ammons et al. 2006), using the upgraded HIRES spectrometer. We measured velocities from the spectral observations using the B96 Doppler technique, with morphed DSTs created with the first iodine observation of each star.

Figure 7 shows a histogram of the velocity rms for our sample. For stars with linear trends and slopes greater than $10 \mathrm{~m} \mathrm{~s}^{-1}$ day $^{-1}$, the scatter is measured with respect to a line fit to the three data points. The majority of the stars $(87 \%)$ had $\mathrm{rms}<10 \mathrm{~m} \mathrm{~s}^{-1}$. The two stars with velocity scatter between 10 and $20 \mathrm{~m} \mathrm{~s}^{-1}$ had predicted velocity "jitter" values in excess of $10 \mathrm{~m} \mathrm{~s}^{-1}$ due to large chromospheric activity indices (Wright 2005). Three stars had $\sigma_{V}>250 \mathrm{~m} \mathrm{~s}^{-1}$, with linear trends indicative of a stellarmass orbital companion, and three stars showed evidence of a short-period planet, with $50<\sigma_{V}<250$. We obtained additional observations of the three planet candidates at Keck Ob-

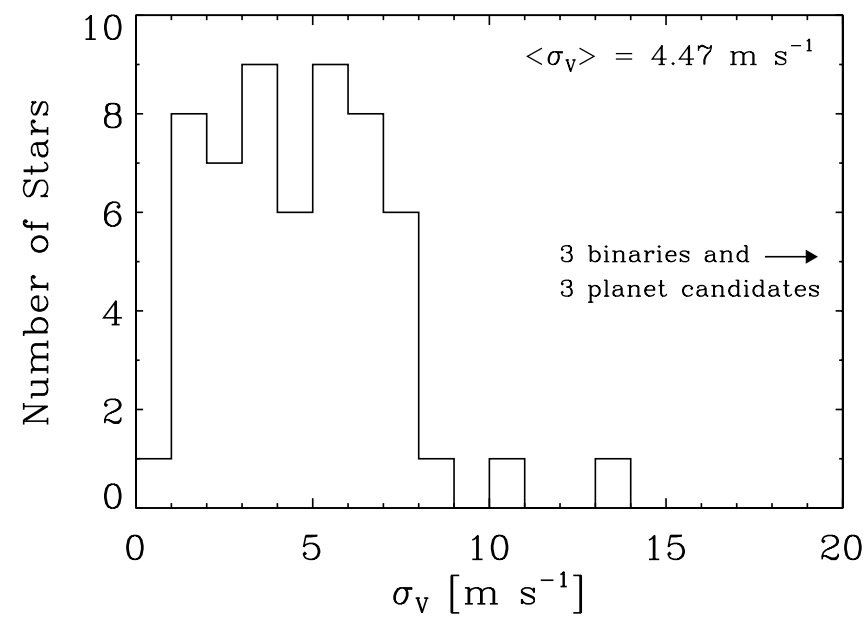

FIG. 7.-Distribution of rms velocities measured for $63 \mathrm{~N} 2 \mathrm{~K}$ target stars. Three binaries (SB1) and three planet candidates with $20 \mathrm{~m} \mathrm{~s}^{-1}<\sigma_{V}<250 \mathrm{~m} \mathrm{~s}^{-1}$ lie outside of the plot range. The two stars with $10 \mathrm{~m} \mathrm{~s}^{-1}<\sigma_{V}<20 \mathrm{~m} \mathrm{~s}^{-1}$ have jitter estimates in excess of $10 \mathrm{~m} \mathrm{~s}^{-1}$. With these 8 outliers removed, the mean is $\left\langle\sigma_{V}\right\rangle=4.47 \mathrm{~m} \mathrm{~s}^{-1}$, with $87 \%$ of the stars having $\sigma_{V}<10 \mathrm{~m} \mathrm{~s}^{-1}$.
TABLE 1

Stellar Parameters

\begin{tabular}{|c|c|c|c|}
\hline Parameter & HD 86081 & HD 224693 & HD 33283 \\
\hline$V \ldots$ & 8.73 & 8.23 & 8.05 \\
\hline$M_{V}$ & 3.93 & 3.36 & 3.36 \\
\hline$B-V$ & 0.664 & 0.639 & 0.641 \\
\hline Spectral type ................. & F8 V & G2 IV & G3 V \\
\hline Distance $(\mathrm{pc}) \ldots \ldots \ldots \ldots$ & 91 & 94 & 86 \\
\hline$[\mathrm{Fe} / \mathrm{H}] \ldots \ldots \ldots$ & $0.257(0.05)$ & $0.343(0.05)$ & $0.366(0.05)$ \\
\hline$T_{\text {eff }}(\mathrm{K}) \ldots \ldots \ldots \ldots \ldots \ldots \ldots$ & $6028(50)$ & $6037(50)$ & $5995(50)$ \\
\hline$V_{\text {rot }} \sin i \mathrm{~km} \mathrm{~s}^{-1} \ldots \ldots \ldots$ & $4.2(0.5)$ & $3.5(0.5)$ & $3.2(0.5)$ \\
\hline $\log g$ & $4.36(0.1)$ & $4.38(0.1)$ & $4.21(0.1)$ \\
\hline$M_{*}\left(M_{\odot}\right) \ldots \ldots \ldots \ldots \ldots$ & $1.21(0.09)$ & $1.33(0.1)$ & $1.24(0.1)$ \\
\hline$R_{*}\left(R_{\odot}\right)$ & $1.22(0.1)$ & $1.70(0.3)$ & $1.20(0.1)$ \\
\hline$S_{\mathrm{HK}}$ & 0.16 & 0.13 & 0.10 \\
\hline $\log R_{\mathrm{HK}}^{\prime}$ & -5.03 & -5.15 & -5.60 \\
\hline$P_{\text {rot }}($ days)........................ & 40.1 & 27.4 & 55.5 \\
\hline
\end{tabular}

servatory, and present here the detection of a $1.5 M_{\mathrm{J}}$ planet in a 2.1 day orbit around HD 86081. The remaining two candidates are still being monitored at Keck.

Excluding the eight stars showing excess variability, the mean velocity rms of our sample is $4.47 \mathrm{~m} \mathrm{~s}^{-1}$. The mean rms for our N2K targets is larger than the short-term test case shown in Figure 5 due to the lower signal-to-noise ratio of the observations $(\mathrm{S} / \mathrm{N} \lesssim 200)$.

However, this performance represents a marked improvement over the previous synthetic template technique used in Paper I, which used the National Solar Observatory (NSO) solar atlas in lieu of our current library of DSTs. Our old method produced a mean velocity scatter of $\sim 15 \mathrm{~m} \mathrm{~s}^{-1}$ and a long tail in the distribution extending to $60 \mathrm{~m} \mathrm{~s}^{-1}$. Of the original sample of 211 N2K targets monitored at Keck beginning in 2004 and analyzed using our older method, 35 were flagged as planet candidates based on having rms scatter $>3 \sigma$ (Paper I). These stars received additional observations, and we performed the Doppler analysis using observed templates to confirm their variability. Three planets from this original sample have been reported previously (Paper I; Fischer et al. 2006). We report here the detection of two new planets from our original sample of Keck targets: a $0.72 M_{\mathrm{J}}$ planet orbiting HD 224693 with a circular 26.7 day period and a Saturn-mass planet in an eccentric 18.2 day orbit around HD 33283.

In the following sections we present the stellar parameters of the host stars, orbital parameters of the planets, and the result of our search for transit events.

\section{1. $H D 86081$}

HD 86081 is an F8 V star with $V=8.73, B-V=0.664$, a Hipparcos parallax-based distance of 91 pc (ESA 1997), and an absolute visual magnitude $M_{V}=3.93$. The star is chromospherically inactive, with no emission seen in the core of the $\mathrm{Ca}$ II $\mathrm{H}$ and $\mathrm{K}$ lines. We measure $S_{\mathrm{HK}}=0.16$ and $\log R_{\mathrm{HK}}^{\prime}=-5.03$ and derive a rotational period of 40.1 days following the calibration of Noyes et al. (1984). Our high-resolution spectral analysis, described in Valenti \& Fischer (2005), yields $T_{\text {eff }}=6028 \mathrm{~K}$, $\log g=4.36,[\mathrm{Fe} / \mathrm{H}]=+0.257$, and $V_{\mathrm{rot}} \sin i=4.2 \mathrm{~km} \mathrm{~s}^{-1}$. Interpolation using the Yi et al. (2001), hereafter $\mathrm{Y}^{2}$, isochrones provided a stellar mass estimate of $1.21 \pm 0.05 M_{\odot}$ and a stellar radius of $1.22 \pm 0.1 R_{\odot}$. The stellar parameters are summarized in Table 1.

We started monitoring HD 86081 in 2005 November, and our Doppler analysis using a morphed template yielded a nonlinear 
TABLE 2

Radial Velocities for HD 86081

\begin{tabular}{|c|c|c|}
\hline $\mathrm{JD}-2,440,000$ & $\begin{array}{c}\mathrm{RV} \\
\left(\mathrm{m} \mathrm{s}^{-1}\right)\end{array}$ & $\begin{array}{l}\text { Uncertainty } \\
\left(\mathrm{m} \mathrm{s}^{-1}\right)\end{array}$ \\
\hline $13694.156 \ldots \ldots$. & -168.30 & 4.20 \\
\hline $13695.155 \ldots \ldots \ldots \ldots \ldots \ldots$ & 129.92 & 4.29 \\
\hline $13697.155 \ldots \ldots \ldots \ldots \ldots \ldots$ & 52.46 & 4.77 \\
\hline $13746.991 \ldots \ldots \ldots \ldots$ & 151.71 & 3.40 \\
\hline $13747.997 \ldots \ldots \ldots \ldots \ldots$ & -184.74 & 3.20 \\
\hline 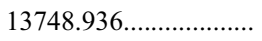 & 201.56 & 2.72 \\
\hline $13749.891 \ldots \ldots \ldots \ldots \ldots$ & -209.26 & 3.06 \\
\hline $13750.907 \ldots \ldots \ldots \ldots \ldots$ & 189.65 & 2.12 \\
\hline $13750.936 \ldots \ldots \ldots \ldots \ldots \ldots$ & 195.23 & 1.85 \\
\hline $13751.883 \ldots \ldots \ldots \ldots \ldots \ldots$ & -170.66 & 2.09 \\
\hline 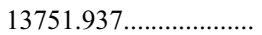 & -196.19 & 3.68 \\
\hline $13752.948 \ldots \ldots \ldots \ldots \ldots \ldots$ & 165.28 & 2.30 \\
\hline $13752.986 \ldots \ldots \ldots \ldots \ldots \ldots$ & 172.90 & 2.22 \\
\hline $13753.060 \ldots \ldots \ldots \ldots \ldots$ & 197.83 & 2.11 \\
\hline $13753.124 \ldots \ldots \ldots \ldots \ldots \ldots$ & 201.11 & 2.25 \\
\hline $13753.912 \ldots \ldots \ldots \ldots \ldots$ & -121.22 & 2.83 \\
\hline $13753.934 \ldots \ldots \ldots \ldots \ldots$ & -132.36 & 2.27 \\
\hline $13753.962 \ldots$ & -142.86 & 1.97 \\
\hline $13754.033 \ldots \ldots \ldots \ldots \ldots$ & -171.86 & 2.17 \\
\hline $13754.069 \ldots \ldots \ldots \ldots \ldots \ldots$ & -185.19 & 2.07 \\
\hline $13775.897 \ldots \ldots \ldots \ldots \ldots \ldots$ & -142.57 & 3.60 \\
\hline 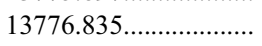 & 180.54 & 3.50 \\
\hline $13777.920 \ldots \ldots \ldots \ldots \ldots$ & -191.64 & 3.46 \\
\hline $13778.927 \ldots \ldots \ldots \ldots \ldots$ & 193.64 & 3.43 \\
\hline $13779.925 \ldots \ldots \ldots \ldots \ldots$ & -207.33 & 3.80 \\
\hline $13781.064 \ldots \ldots \ldots \ldots \ldots \ldots$ & 190.15 & 3.64 \\
\hline
\end{tabular}

velocity variation with $\mathrm{rms}=154 \mathrm{~m} \mathrm{~s}^{-1}$ from the first three measurements. Additional monitoring revealed a clear 2.1375 day periodicity. We list our velocity measurements in Table 2 along with the Julian Dates and internal measurement uncertainties. We report here only the velocities measured using a high-quality observed template, due to their higher velocity precision.

In order to search for a the best-fit orbital solution, we augmented the internal measurements errors by adding in quadrature a photometric jitter estimate of $3.7 \mathrm{~m} \mathrm{~s}^{-1}$ based on the chromospheric activity index of the star (Wright 2005). Figure 8 shows

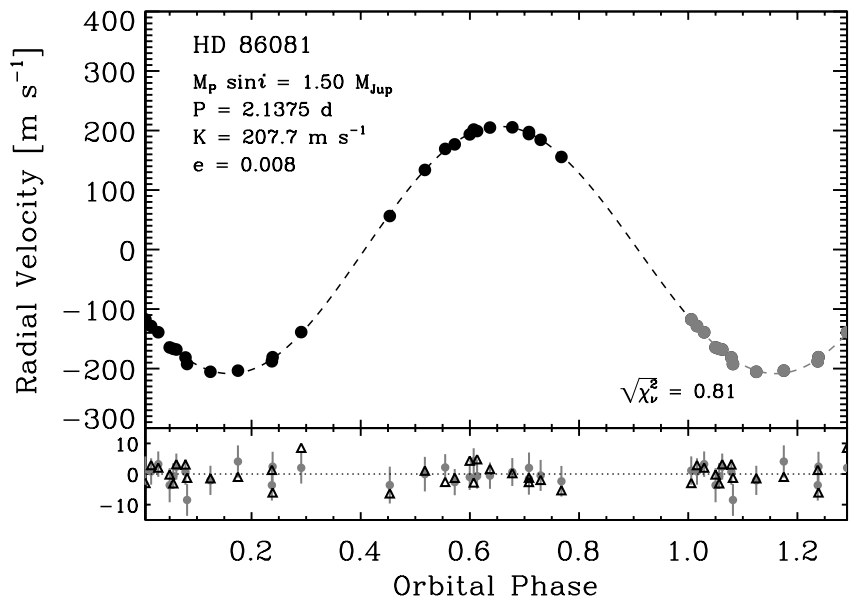

FIG. 8.-Top: Phased radial velocity measurements of HD 86081 (observed template). The dashed line shows the best-fit orbital solution. Bottom: The residuals of the orbital fit ( filled circles) and the difference between the velocities measured with observed and morphed templates (open triangles). The rms of the fit residuals is $3.20 \mathrm{~m} \mathrm{~s}^{-1}$ and the rms difference between the two sets of RV measurements is $2.8 \mathrm{~m} \mathrm{~s}^{-1}$.
TABLE 3

Orbital Parameters for HD 86081 from Observed and Morphed Templates

\begin{tabular}{|c|c|c|}
\hline Parameter & Observed & Morphed \\
\hline$P($ days $) .. . \ldots \ldots \ldots$ & $2.1375(0.0002)$ & $2.1378(0.0002)$ \\
\hline$T_{p}^{\mathrm{a}}(\mathrm{JD}) \ldots \ldots \ldots \ldots \ldots \ldots$ & $2453694.8(0.3)$ & $2453694.4(0.4)$ \\
\hline e......................... & $0.008(0.004)$ & $0.007(0.005)$ \\
\hline 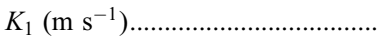 & $207.7(0.8)$ & $207.8(1.0)$ \\
\hline$\omega(\operatorname{deg}) \ldots \ldots \ldots \ldots \ldots \ldots \ldots \ldots$ & $251(40)$ & $192(70)$ \\
\hline 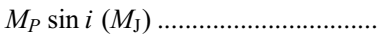 & 1.50 & 1.50 \\
\hline$a(\mathrm{AU})$ & 0.035 & 0.035 \\
\hline Fit $\mathrm{rms}\left(\mathrm{m} \mathrm{s}^{-1}\right) \ldots \ldots$ & 3.20 & 4.38 \\
\hline 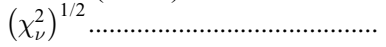 & 0.81 & 0.87 \\
\hline 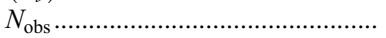 & 26 & 26 \\
\hline
\end{tabular}

a Time of periastron passage.

the phased RVobservations of HD 86081 together with the bestfit orbit solution: $P=2.1375$ days, $K=207.7 \mathrm{~km} \mathrm{~s}^{-1}$, and $e=0.008 \pm 0.004$. From the orbital parameters and adopted stellar mass of $1.21 M_{\odot}$, we derive a minimum planet mass $M_{P} \sin i=1.50 M_{\mathrm{J}}$ and semimajor axis $a=0.035 \mathrm{AU}$. The lower panel of Figure 8 shows the fit residuals ( filled circles), which have an rms scatter of $3.20 \mathrm{~m} \mathrm{~s}^{-1}$ and $\left(\chi_{\nu}^{2}\right)^{1 / 2}=0.81$. Also shown in the lower panel are the differences between the measurements made with morphed and observed templates (open triangles), which show excellent agreement $\left(\mathrm{rms}=2.8 \mathrm{~m} \mathrm{~s}^{-1}\right)$.

We list the best-fit orbital parameters in Table 3 along with the parameters determined from the morphed-template velocities. The two sets of parameters are identical within errors. The parameter uncertainties were estimated using a Monte Carlo method. For each of 1000 trials, the fit was subtracted from the measurements, the scrambled residuals were added back to the measurements, and a new solution was determined. The standard

TABLE 4

Radial Velocities for HD 224693

\begin{tabular}{|c|c|c|}
\hline $\mathrm{JD}-2,440,000$ & $\begin{array}{c}\mathrm{RV} \\
\left(\mathrm{m} \mathrm{s}^{-1}\right)\end{array}$ & $\begin{array}{c}\text { Uncertainty } \\
\left(\mathrm{m} \mathrm{s}^{-1}\right)\end{array}$ \\
\hline $13191.097 \ldots$ & 47.16 & 5.09 \\
\hline 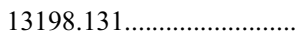 & 20.71 & 5.54 \\
\hline 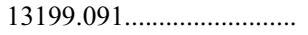 & 17.74 & 5.50 \\
\hline $13200.042 \ldots \ldots \ldots \ldots \ldots \ldots$ & 9.35 & 5.06 \\
\hline $13367.705 \ldots \ldots \ldots \ldots \ldots \ldots \ldots$ & -37.79 & 2.65 \\
\hline $13368.713 \ldots \ldots \ldots \ldots \ldots \ldots$ & -26.67 & 3.26 \\
\hline $13369.714 \ldots \ldots \ldots \ldots \ldots \ldots$ & -26.54 & 2.50 \\
\hline $13370.736 \ldots \ldots \ldots \ldots \ldots \ldots \ldots$ & -13.68 & 11.3 \\
\hline $13550.112 \ldots \ldots \ldots \ldots \ldots \ldots \ldots \ldots$ & -20.60 & 3.48 \\
\hline $13551.122 \ldots \ldots$. & -18.08 & 3.25 \\
\hline $13552.090 \ldots \ldots \ldots \ldots \ldots \ldots$ & -27.37 & 3.38 \\
\hline $13571.088 \ldots \ldots \ldots \ldots \ldots \ldots \ldots$ & 32.34 & 3.31 \\
\hline $13603.089 \ldots \ldots \ldots \ldots \ldots \ldots$ & -15.32 & 3.06 \\
\hline $13604.062 \ldots \ldots \ldots \ldots \ldots \ldots \ldots$ & -21.39 & 2.87 \\
\hline 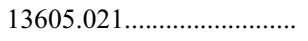 & -24.05 & 2.87 \\
\hline 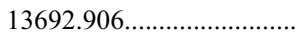 & -6.92 & 1.97 \\
\hline $13693.850 \ldots \ldots \ldots \ldots \ldots \ldots$ & -3.39 & 1.95 \\
\hline $13694.762 \ldots \ldots \ldots \ldots \ldots \ldots$ & 3.36 & 2.11 \\
\hline 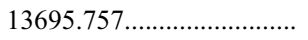 & 9.33 & 2.09 \\
\hline $13696.751 \ldots \ldots \ldots \ldots \ldots \ldots \ldots \ldots$ & 23.32 & 2.01 \\
\hline $13723.810 \ldots \ldots \ldots \ldots \ldots \ldots \ldots \ldots$ & 31.15 & 2.65 \\
\hline $13724.756 \ldots \ldots \ldots \ldots$ & 35.05 & 2.68 \\
\hline $13746.696 \ldots \ldots \ldots$ & -6.67 & 3.14 \\
\hline $13752.761 \ldots \ldots \ldots \ldots \ldots \ldots \ldots \ldots \ldots$ & 40.52 & 2.67 \\
\hline
\end{tabular}




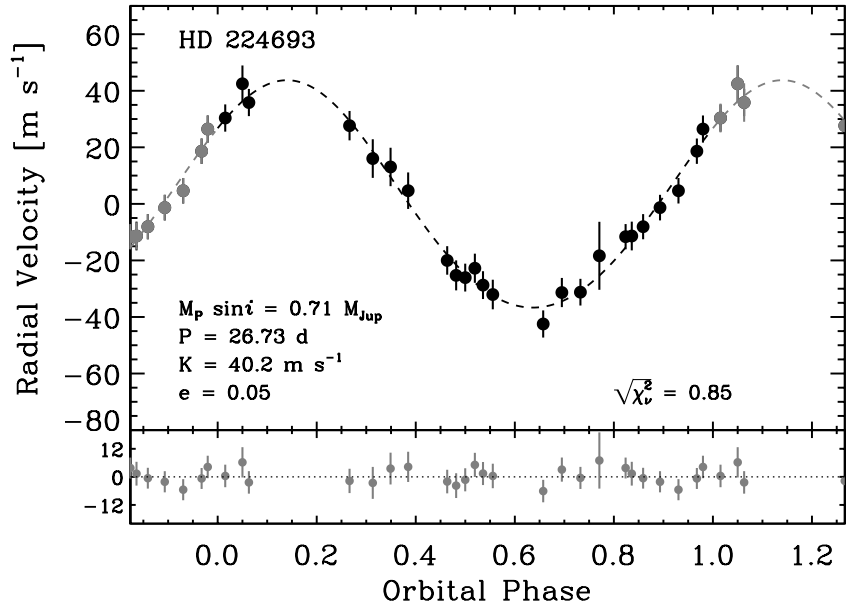

FIG. 9.- Phased radial velocity measurements of HD 224693 and best-fit orbital solution. The fit residuals have an rms of $4.07 \mathrm{~m} \mathrm{~s}^{-1}$.

deviations of the parameters derived from all trials were adopted as the $1 \sigma$ uncertainties.

\subsection{HD 224693}

HD 224693 is a $V=8.23$, G2 IV star with $B-V=0.639$, a parallax-based distance of $94 \mathrm{pc}$, and $M_{V}=3.36$ (ESA 1997). The star is chromospherically quiet, with $\log R_{\mathrm{HK}}^{\prime}=-5.15$ and a rotational period of 27.4 days, based on the calibration by Noyes et al. (1984). Our spectral synthesis modeling yields $T_{\text {eff }}=$ $6037 \mathrm{~K}, \log g=4.38, V_{\text {rot }} \sin i=3.5 \mathrm{~km} \mathrm{~s}^{-1}$, and $[\mathrm{Fe} / \mathrm{H}]=$ +0.343 . The mass of the star derived from $\mathrm{Y}^{2}$ isochrones ( $\mathrm{Yi}$ et al. 2001) is estimated to be $1.33 \pm 0.1 M_{\odot}$ with a radius of $1.70 \pm 0.3 R_{\odot}$.

HD 224693 was observed at Keck as part of the N2K program beginning in 2004 July. The first four Doppler observations showed a modest velocity rms of $16 \mathrm{~m} \mathrm{~s}^{-1}$. At that time, the synthetic template algorithm was still being tested, so a standard template was made to confirm the low-amplitude variation. We obtained a total of 24 radial velocities, listed in Table 4 . We augmented the internal uncertainties with a jitter estimate of $4.0 \mathrm{~m} \mathrm{~s}^{-1}$ based on its chromospheric activity index (Wright 2005).

The velocities are well fit by a Keplerian with $P=26.73$ days, $K=40.2 \mathrm{~m} \mathrm{~s}^{-1}$, and eccentricity $e=0.05 \pm 0.03$. Figure 9 shows the phased radial velocity measurements along with the best-fit Keplerian model. The rms fit to the model is $4.07 \mathrm{~m} \mathrm{~s}^{-1}$ with $\left(\chi_{\nu}^{2}\right)^{1 / 2}=0.85$, consistent with the measurement errors. From our orbital parameters and the adopted stellar mass of $1.33 M_{\odot}$

TABLE 5

Orbital Parameters For HD 224693 and HD 33283

\begin{tabular}{|c|c|c|}
\hline Parameter & HD 224693 & HD 33283 \\
\hline$P$ (days) & $26.73(0.02)$ & $18.179(0.007)$ \\
\hline$T_{p}^{\mathrm{a}}(\mathrm{JD})$ & 2453193.9 (3.) & $2453017.6(0.3)$ \\
\hline e & $0.05(0.03)$ & $0.48(0.05)$ \\
\hline$K_{1}\left(\mathrm{~m} \mathrm{~s}^{-1}\right) \ldots \ldots$ & $40.2(2)$. & $25.2(2)$. \\
\hline$\omega(\operatorname{deg})$ & 6. (200) & $155.8(8)$. \\
\hline$M_{P} \sin i\left(M_{\mathrm{J}}\right) \ldots \ldots \ldots \ldots \ldots \ldots \ldots \ldots \ldots \ldots \ldots \ldots \ldots$ & 0.71 & 0.33 \\
\hline$a(\mathrm{AU})$ & 0.192 & 0.145 \\
\hline Fit rms $\left(\mathrm{m} \mathrm{s}^{-1}\right)$ & 4.07 & 3.60 \\
\hline$\left(\chi_{\nu}^{2}\right)^{1 / 2}$ & 0.85 & 0.77 \\
\hline$N_{\text {obs }}$ & 24 & 25 \\
\hline
\end{tabular}

${ }^{\text {a }}$ Time of periastron passage.
TABLE 6

Radial Velocities for HD 33283

\begin{tabular}{|c|c|c|}
\hline $\mathrm{JD}-2,440,000$ & $\begin{array}{c}\mathrm{RV} \\
\left(\mathrm{m} \mathrm{s}^{-1}\right)\end{array}$ & $\begin{array}{l}\text { Uncertainty } \\
\left(\mathrm{m} \mathrm{s}^{-1}\right)\end{array}$ \\
\hline $13014.852 \ldots \ldots \ldots$ & 10.57 & 5.45 \\
\hline $13015.858 \ldots \ldots \ldots \ldots \ldots \ldots$ & 1.88 & 5.35 \\
\hline $13016.850 \ldots \ldots \ldots \ldots \ldots \ldots$ & -11.57 & 4.98 \\
\hline $13071.767 \ldots \ldots \ldots \ldots \ldots$ & -29.19 & 4.85 \\
\hline $13072.843 \ldots \ldots \ldots \ldots \ldots$ & -31.26 & 5.07 \\
\hline $13368.955 \ldots \ldots \ldots \ldots \ldots$ & 4.10 & 4.94 \\
\hline $13369.808 \ldots \ldots \ldots \ldots \ldots$ & 4.87 & 4.99 \\
\hline $13397.796 \ldots \ldots \ldots \ldots \ldots \ldots$ & -8.86 & 4.52 \\
\hline $13398.870 \ldots \ldots \ldots \ldots \ldots \ldots$ & -20.06 & 5.14 \\
\hline $13428.792 \ldots \ldots \ldots \ldots \ldots \ldots$ & 17.14 & 4.50 \\
\hline $13605.139 \ldots \ldots \ldots \ldots \ldots \ldots$ & 10.66 & 4.35 \\
\hline $13692.917 \ldots \ldots \ldots \ldots \ldots$ & -12.34 & 3.93 \\
\hline $13693.979 \ldots \ldots \ldots \ldots \ldots \ldots$ & -2.42 & 4.23 \\
\hline $13694.975 \ldots \ldots \ldots \ldots \ldots \ldots$ & -0.03 & 4.35 \\
\hline 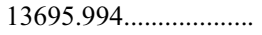 & 6.55 & 4.33 \\
\hline $13696.981 \ldots \ldots \ldots \ldots \ldots . .$. & 9.04 & 4.26 \\
\hline $13723.925 \ldots \ldots \ldots \ldots \ldots \ldots$ & 9.44 & 4.49 \\
\hline $13724.932 \ldots \ldots \ldots \ldots \ldots \ldots$ & 4.19 & 4.53 \\
\hline $13746.852 \ldots \ldots \ldots \ldots \ldots \ldots$ & -15.10 & 4.81 \\
\hline $13747.891 \ldots \ldots \ldots \ldots \ldots \ldots$ & -2.94 & 4.60 \\
\hline $13748.809 \ldots \ldots \ldots \ldots \ldots \ldots$ & -0.13 & 4.37 \\
\hline $13749.824 \ldots \ldots \ldots \ldots \ldots \ldots$ & 5.66 & 4.27 \\
\hline $13750.822 \ldots \ldots \ldots \ldots \ldots$ & 12.91 & 4.14 \\
\hline $13751.875 \ldots \ldots \ldots \ldots \ldots \ldots$ & 14.61 & 3.34 \\
\hline $13752.878 \ldots \ldots \ldots \ldots \ldots \ldots$ & 13.17 & 3.89 \\
\hline
\end{tabular}

we derive a minimum planet mass $M_{P} \sin i=0.71 M_{\mathrm{J}}$ and semimajor axis $a=0.192 \mathrm{AU}$. The full list of derived orbital parameters is given in Table 5 .

\subsection{HD 33283}

HD 33283 is a $V=8.05$, G3 V star with $B-V=0.641$. Based on the Hipparcos parallax, it has a distance of $86 \mathrm{pc}$ and absolute visual magnitude $M_{V}=3.36$ (ESA 1997). The star is chromospherically inactive with $S=0.10, \log R_{\mathrm{HK}}^{\prime}=-5.60$, and a predicted rotational period of 55.5 days. Spectral synthesis modeling yields $T_{\text {eff }}=5995 \mathrm{~K}, \log g=4.21, V_{\text {rot }} \sin i=3.2 \mathrm{~km} \mathrm{~s}^{-1}$, and $[\mathrm{Fe} / \mathrm{H}]=+0.366$. Interpolation of the $\mathrm{Y}^{2}$ isochrones ( $\mathrm{Yi}$ et al. 2001) results in a mass of $1.24 \pm 0.1 M_{\odot}$ and radius of $1.20 \pm$ $0.1 R_{\odot}$. We summarize the stellar characteristics in Table 1 .

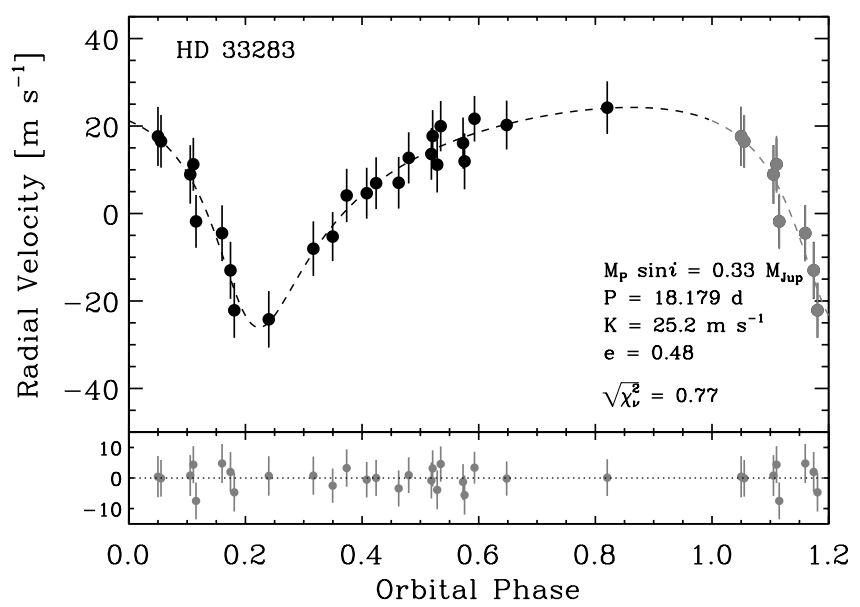

FIG. 10.-Phased radial velocity measurements of HD 33283 and best-fit orbital solution. The fit residuals have an rms of $3.60 \mathrm{~m} \mathrm{~s}^{-1}$. 
TABLE 7

Photometric Observations of HD 86081 AND HD 224693

\begin{tabular}{|c|c|}
\hline $\begin{array}{l}\text { Observation Date } \\
(\mathrm{HJD}-2,400,000)\end{array}$ & $\begin{array}{l}\Delta(b+y) / 2 \\
\quad(\mathrm{mag})\end{array}$ \\
\hline \multicolumn{2}{|l|}{ HD 86081} \\
\hline $53,739.9097 \ldots \ldots \ldots . .$. & 1.2972 \\
\hline 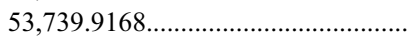 & 1.2987 \\
\hline 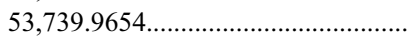 & 1.2985 \\
\hline 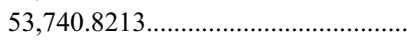 & 1.2976 \\
\hline 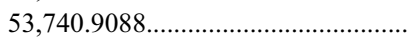 & 1.2964 \\
\hline
\end{tabular}

Note-Table 7 is published in its entirety in the electronic edition of the Astrophysical Journal. A portion is shown here for guidance regarding its form and content.

We began monitoring HD 33283 at Keck in 2004 July. The first five radial velocities showed a modest rms of $21 \mathrm{~m} \mathrm{~s}^{-1}$, with a $17 \mathrm{~m} \mathrm{~s}^{-1}$ change between the third and fourth observation. At that time, our synthetic template algorithm was still being tested, so a standard observed template was obtained to confirm the variations. The 25 velocities measured with the observed template are listed in Table 6 along with the internal measurement uncertainties. Based on the chromospheric emission index, we expect $4.0 \mathrm{~m} \mathrm{~s}^{-1}$ of stellar jitter, which we added in quadrature to the internal uncertainties.

The best-fit Keplerian orbit solution yields $K=25.2 \mathrm{~m} \mathrm{~s}^{-1}$, $P=18.179$ days, and $e=0.48$. The phased radial velocities and orbit solution are shown in Figure 10. The fit has rms $=3.60 \mathrm{~m} \mathrm{~s}^{-1}$ and $\left(\chi_{\nu}^{2}\right)^{1 / 2}=0.77$, consistent with the measurement errors. From our adopted stellar mass and best-fit orbit parameters, we estimate a minimum planet mass of $0.33 M_{\mathrm{J}}$ and $a=0.145 \mathrm{AU}$. The full orbit solution is summarized in Table 5.

\subsection{Photometry of the Host Stars}

Between 2005 November and 2006 February, we obtained high-precision photometry of two of the three planetary host stars in this paper with the T8 and T10 $0.8 \mathrm{~m}$ APTs at Fairborn Observatory. The APTs can detect short-term, low-amplitude brightness variability in the stars due to rotational modulation in the visibility of magnetic surface features such as spots and plages (e.g., Henry et al. 1995), as well as longerterm variations associated with stellar magnetic cycles (Henry 1999). The photometric observations can help to establish whether observed radial velocity variations are caused by stellar activity or planetary-reflex motion (e.g., Henry et al. 2000a). Queloz et al. (2001) and Paulson et al. (2004) have found several examples of periodic radial velocity variations in solar-type stars caused by photospheric spots and plages. The APT observations are also useful to search for possible transits of the planetary companions (e.g., Henry et al. 2000b; Sato et al. 2005b).
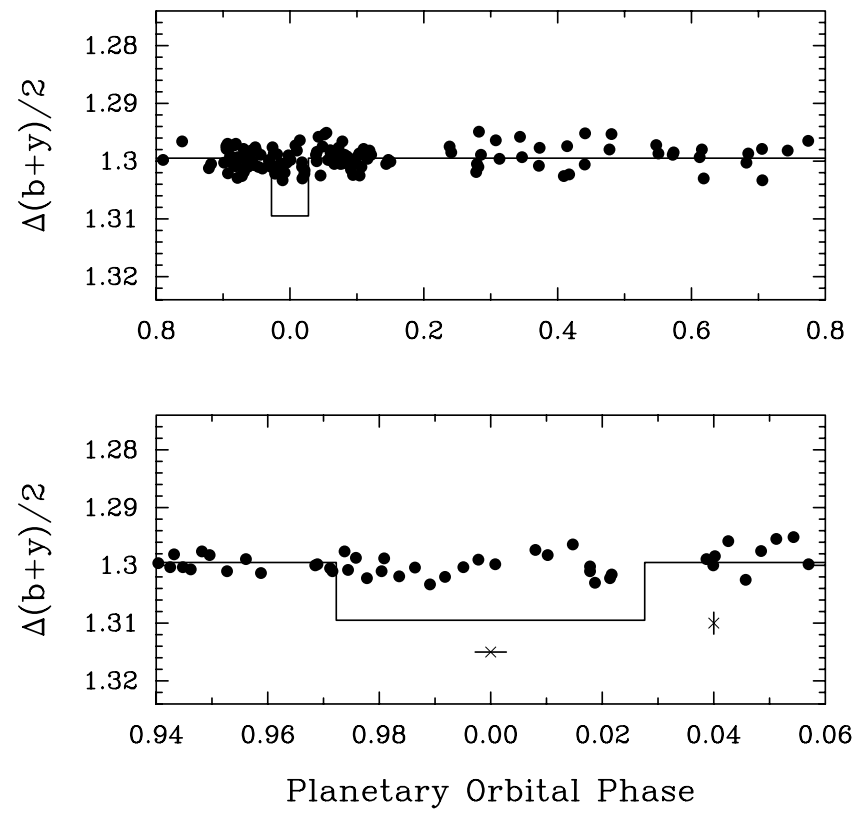

FIG. 11.-Top: Complete set of Strömgren $(b+y) / 2$ photometric observations of HD 86081 obtained with the T8 $0.8 \mathrm{~m}$ APT at Fairborn Observatory and plotted against orbital phase of the planetary companion. The predicted time, depth, and duration of possible transits are shown schematically. The star exhibits no optical variability on the radial velocity period larger than $0.0004 \mathrm{mag}$ or so. Bottom: Observations around the predicted time of transit are replotted with an expanded scale on the abscissa. The error bars are described in the text. Transits deeper than $0.001 \mathrm{mag}$ or so are ruled out by these observations.

The T8 and T10 APTs are both equipped with two-channel precision photometers, each employing two EMI 9124QB bialkali photomultiplier tubes to make simultaneous measurements in the Strömgren $b$ and $y$ passbands. The APTs measure the difference in brightness between a program star and a nearby comparison star. The local comparison stars used for the program stars were HD 84664 and HD 203 for HD 86081 and HD 224693, respectively. Strömgren $b$ and $y$ differential magnitudes were computed and corrected for differential extinction with nightly extinction coefficients and transformed to the Strömgren system with yearly mean transformation coefficients. The external precision of the differential magnitudes is typically between 0.0012 and $0.0017 \mathrm{mag}$ for these telescopes, as determined from observations of pairs of constant stars. Finally, we combined the Strömgren $b$ and $y$ differential magnitudes into a single $(b+y) / 2$ passband to maximize the precision of the photometric measurements. The individual photometric observations of HD 86081 and HD 224693 are given in Table 7. Further information on the automatic telescopes, photometers, observing procedures, and data reduction techniques can be found in Henry (1999) and Eaton et al. (2003).

The photometric results for the two stars we measured are summarized in Table 8 . We have no photometry as yet on HD

TABLE 8

Photometric Results for the Planetary Host Stars

\begin{tabular}{|c|c|c|c|c|c|c|}
\hline $\begin{array}{l}\text { Star } \\
(1)\end{array}$ & $\begin{array}{c}\text { Date Range } \\
\text { (HJD }-2,450,000) \\
(2)\end{array}$ & $\begin{array}{c}N_{\text {obs }} \\
\text { (3) }\end{array}$ & $\begin{array}{c}\sigma \\
(\mathrm{mag}) \\
(4)\end{array}$ & $\begin{array}{l}\text { Semiamplitude } \\
\text { (mag) } \\
\text { (5) }\end{array}$ & $\begin{array}{c}\text { Transit Probability } \\
\text { (\%) } \\
(6)\end{array}$ & $\begin{array}{c}\text { Transits } \\
\text { (7) }\end{array}$ \\
\hline 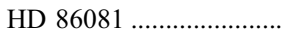 & $3739-3777$ & 135 & 0.0019 & $0.0004 \pm 0.0002$ & 17.4 & No \\
\hline 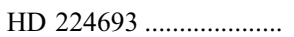 & $3691-3733$ & 22 & 0.0018 & $0.0003 \pm 0.0004$ & 4.2 & $?$ \\
\hline
\end{tabular}



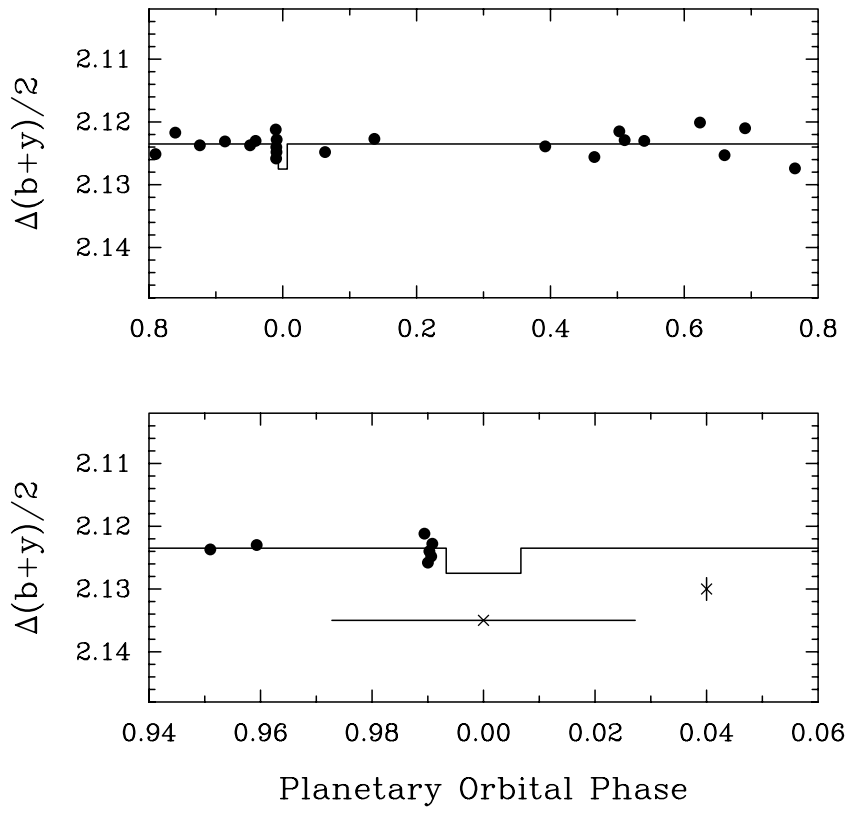

FIG. 12.-Top: Complete set of Strömgren $(b+y) / 2$ photometric observations of HD 224693 obtained with the T10 $0.8 \mathrm{~m}$ APT at Fairborn Observatory and plotted against orbital phase of the planetary companion. The predicted time, depth, and duration of possible transits are shown schematically. The star exhibits no optical variability on the radial velocity period larger than $0.0003 \mathrm{mag}$ or so. Bottom: Observations around the predicted time of transit are replotted with an expanded scale on the abscissa. The error bars are described in the text. Our observations are too few to rule out possible transits.

33283. Columns (2) and (3) give the date range of the photometric observations and the total number of individual differential magnitudes, respectively. The standard deviations in column (4) refer to the spread of the $(b+y) / 2$ measurements around the mean values of the data sets. All standard deviations are consistent with the constancy of the host stars and their comparison stars. Periodogram analyses of the two data sets do not reveal any significant periodicities. We computed the semiamplitudes of the light curves (col. [5]) with least-squares sine fits of the observations phased to the radial velocity periods. The resulting amplitudes are both 0.0004 mag or below; this absence of light variability on the radial velocity periods supports planetary-reflex motion as the cause of the radial velocity variations in both stars. Although we have no photometry of HD 33283, it is the most inactive of the three stars (Table 1) and so should show a similar lack of photometric variability.

The sixth column of Table 8 gives the geometric probability of transits for the three systems we observed photometrically, computed from equation (1) of Seagroves et al. (2003) and assuming random orbital inclinations. The final column gives the results of our search for planetary transits of the two companions as shown in Figures 11 and 12 and discussed below.

The top panels in Figures 11 and 12 plot the complete photometric data set for each star against the planetary orbital phase, computed from the orbital elements in Tables 3 and 5; zero phase in each case refers to a time of inferior conjunction (midtransit). The solid curve in each panel approximates the predicted transit light curve, assuming a planetary orbital inclination of $90^{\circ}$ (central transits). The out-of-transit light level corresponds to the mean brightness of the observations. The transit durations are calculated from the orbital elements, while the transit depths are derived from the estimated stellar radii (Table 1) and planetary radii computed with the models of Bodenheimer et al. (2003). The bottom panel of each figure shows the observations around the predicted time of midtransit replotted with an expanded scale on the abscissa. The horizontal error bar below each predicted transit curve represents the approximate $\pm 1 \sigma$ uncertainty in the time of mid transit, based on Monte Carlo simulations and the uncertainties in the orbital elements. The vertical error bar represents the precision of the measurements in each case.

Our photometric observations of HD 86081 cover the predicted transit window quite well. The 21 observations within the transit window in the bottom panel of Figure 11 have a mean brightness level of $1.3003 \pm 0.0004 \mathrm{mag}$. The 114 observations outside the transit window have a mean of $1.2993 \pm 0.0002 \mathrm{mag}$. Thus, the two values agree to within $0.001 \mathrm{mag}$, or $\pm 2 \sigma$, placing very shallow limits on possible transits. In the case of HD 224693 , our data around the predicted time of transit are far to sparse to rule out possible transits at this time (Fig. 12) and we are continuing to monitor this star.

\section{SUMMARY AND DISCUSSION}

We have presented a modification to the $\mathrm{B} 96$ precision radial velocity technique that eliminates the need to obtain template observations for each target star. We replace observed templates with synthetic versions derived by modifying the deconvolved stellar template (DST) of a star with similar characteristics as the target star. The depths and widths of the spectral lines in the surrogate DST are modified until, when multiplied by an iodine template and convolved with a model of the spectrometer PSF, a fit to the target star's observed star-plus-iodine spectrum is obtained. Based on our tests of Keck HIRES spectra of a set of chromospherically inactive stars, we have determined that morphed templates yield velocity measurements with precision within a factor of 2 of those produced by observed templates, with a short-term precision of $\sim 3 \mathrm{~m} \mathrm{~s}^{-1}$ and long-term stability within $5 \mathrm{~m} \mathrm{~s}^{-1}$.

While observed templates yield the highest velocity precision, the observational time savings afforded by morphed DSTs make them favorable alternatives for many types of planet search programs. Examples include surveys containing evolved stars such as K giants, where stellar jitter is the dominant noise source (Frink et al. 2002; Sato et al. 2005a); surveys conducted on small telescopes where it is not possible to obtain high enough signal-tonoise ratios for template observations; testing for binarity among SIM grid star candidates (Frink et al. 2001; Gould 2001); and surveys containing faint stars such as $\mathrm{M}$ dwarfs.

Morphed templates are also ideal for the "Next 2000 Stars" (N2K) planet search, which uses a quick-look observing strategy to efficiently search for short-period planets within a large sample of stars (Paper I). Synthetic templates are an integral part of the N2K observing strategy and have already yielded important results. We report here the discovery of three new planets from the Keck N2K program: a $1.50 M_{\mathrm{J}}$ planet in a 2.1375 day orbit around HD 86081; a Saturn-mass planet in an eccentric, 18.179 day orbit around HD 33283; and a Jovian-mass planet with a circular, 26.73 day orbit around HD 224693.

The quick-look strategy used by the N2K consortium involves observing stars during three consecutive nights, and Monte Carlo simulations have shown that the strategy is most sensitive to planets with $M_{P} \sin i>0.5 M_{\mathrm{J}}$ and periods between 1.2 and 14 days (Paper I). However, the planets orbiting HD 33283 and HD 224693 have orbital periods significantly longer than 14 days. These detections were aided by the fact that intensive follow-up observations were not initiated until after the fourth or fifth observations were obtained. These two cases serve as important illustrations of how the sensitivity of the N2K quick-look observing strategy can be greatly enhanced with additional observations and an increased time baseline. 
The 2.1375 day orbital period of HD $86081 \mathrm{~b}$ bridges the gap between the 3 day pileup seen in the period distribution of hot Jupiters (HJs; 3 days $\leqslant P<15$ days; Butler et al. 2006; Udry et al. $2003)$ discovered by RV surveys, and the extremely short period ( $P \lesssim 2$ days) planets found by the Optical Gravitational Lensing Experiment planet transit search (OGLE; Udalski et al. 2002; Konacki et al. 2003; Bouchy et al. 2004). While these "very hot jupiters" (VHJs) are common among the OGLE planets, comprising three out of five transit detections, Doppler surveys have previously found only two other Jovian-mass planets with a period significantly less than 3 days (HD 73256, $P=2.55$ days and HD 189733, $P=2.22$ days; Udry et al. 2003; Bouchy et al. 2005). Gaudi, Seager. \& Mallen-Ornelas (2005, hereafter GSM05) showed that this apparent inconsistency between RV and transit searches can be reconciled by considering the different selection biases for the two search methods and the $P^{-5 / 2}$ sensitivity scaling for ground-based transit searches. By accounting for these effects, GSM05 concluded that VHJs represent a rare class of exoplanet, with an occurrence rate only $10 \%$ that of HJs, based on the aggregate sample from RV and transit searches.

Since RV surveys such as the California and Carnegie Planet Search (CCPS) and N2K are complete down to Saturn-mass planets with orbital periods less than 15 days $\left(K=25 \mathrm{~m} \mathrm{~s}^{-1}\right)$, we can use the combined sample of planets from these two surveys to estimate the relative number of VHJs and HJs. Out of $\sim 1800$ total stars, these two RV surveys have yielded $16 \mathrm{HJs}$ and two VHJs (using a somewhat arbitrary lower cutoff at $P=3$ days and therefore including HD 83443 as done by GSM05). Thus, we estimate the ratio of HJs to VHJs is $\sim 8$, which is in quantitative agreement with the estimates of GSM05. A much more detailed analysis will be provided in a future publication in the N2K Consortium series (D. A. Fischer \& G. Laughlin 2006, in preparation).

The relative paucity of VHJs provides an interesting puzzle, and the answer is likely related to the way in which close-in giant planets are formed and how they interacted with their early circumstellar environments. One of the leading theories suggests that giant planets migrate inward along nearly circular orbits through interactions with their circumstellar disks. These interactions can take the form of gravitational scattering and physical collisions with planetesimals in the remnant disk (Murray et al. 1998), or through type I or II orbital migration (Trilling et al. 1998; Ida \& Lin 2004). These disk interaction scenarios predict very few high-mass planets $\left(M_{P} \gtrsim 2 M_{\mathrm{J}}\right)$ at small semimajor axes due to mass loss suffered from Roche lobe overflow. This prediction is in accordance with the observed deficit of high-mass, short-period planets.

There is another, very different theory for forming short-period giant planets. In this alternate scenario, planets with initially high eccentricity and small periastron passages become tidally circularized at small orbital separations. Using the observed properties of extrasolar planets, Ford \& Rasio (2006) showed that there exists a sharp cutoff in the mass-period distribution nearly equal to twice the Roche limit, corresponding to the ideal circularization distance (Faber et al. 2005; Gu et al. 2003; Rasio \& Ford 1996). If the original eccentricity is induced by planet-planet or planet-star interactions (e.g., Weidenschilling \& Marzari 1996; Rasio \& Ford 1996), then the expectation is that there would be fewer highmass HJs due to their inertial resistance to scattering, similar to the migration scenario and also in agreement with the observed data.

The Roche limit scales inversely with planet-star mass ratio and proportionally with the planet radius. Since the planet radius is nearly constant for $M_{P}>1 M_{\mathrm{J}}$, the tidal circularization theory helps explain the rarity of massive planets in extremely short-period orbits. Indeed, the two most recently discovered VHJs, HD $189733 \mathrm{~b}$ and HD $86081 \mathrm{~b}$, lie safely beyond the ideal circularization radius. However, Ford \& Rasio (2006) caution that the distance of the cutoff was derived under the assumption that the slope follows the Roche limit since the slope cannot be constrained by the current observational data. Additional leverage can be gained by increasing the number of targets monitored in order to find rare high-mass planets close to the circularization limit, or increasing precision in order to find low-mass, shortperiod systems. This highlights the importance of programs like N2K that are specifically designed to efficiently search for shortperiod planets within a large sample of stars.

We would like to thank Ansgar Reiners for his constructive comments and suggestions on our spectral morphing technique. Thanks to Tim Robishaw for lending his expertise in data presentation, and for his many useful IDL Postscript and plotting routines. We gratefully acknowledge the efforts and dedication of the Keck Observatory staff. We thank the NOAO and NASA telescope assignment committees for generous allocations of telescope time. We appreciate funding from NASA grant NNG05GK92G (to G. W. M.) for supporting this research. D. A. F. is a Cottrell Science Scholar of the Research Corporation and acknowledges support from NASA grant NNG05G164G that made this work possible. We also thank NSF for its grants AST-0307493 and AST-9988358 (to S. S. V.). G. W. H. acknowledges support from NASA grant NCC5-511 and NSF grant HRD 97-06268. The authors wish to extend special thanks to those of Hawaiian ancestry on whose sacred mountain of Mauna Kea we are privileged to be guests. Without their generous hospitality, the Keck observations presented herein would not have been possible.

\section{REFERENCES}

Ammons, S. M., Robinson, S. E., Strader, J., Laughlin, G., Fischer, D., \& Wolf, A. 2006, ApJ, 638, 1004

Bodenheimer, P., Laughlin, G., \& Lin, D. N. C. 2003, ApJ, 592, 555

Bouchy, F., Pont, F., Santos, N. C., Melo, C., Mayor, M., Queloz, D., \& Udry, S. 2004, A\&A, 421, L13

Bouchy, F., et al. 2005, A\&A, 444, L15

Butler, R. P., Marcy, G. W., Williams, E., McCarthy, C., Dosanjh, P., \& Vogt, S. S. 1996, PASP, 108, 500 (B96)

Butler, R. P., et al. 2006, ApJ, in press

Eaton, J. A., Henry, G. W., \& Fekel, F. C. 2003, in The Future of Small Telescopes in the New Millennium, Vol. 2 The Telescopes We Use, ed. T.D. Oswalt (Dordrecht: Kluwer), 189

ESA. 1997, The Hipparcos and Tycho Catalogues (ESA SP-1200; Noordwijk: ESA)

Faber, J. A., Rasio, F. A., \& Willems, B. 2005, Icarus, 175, 248

Fischer, D. A., et al. 2005, ApJ, 620, 481 (Paper I) 2006, ApJ, 637, 1094

Ford, E. B., \& Rasio, F. A. 2006, ApJ, 638, L45

Frink, S., Mitchell, D. S., Quirrenbach, A., Fischer, D. A., Marcy, G. W., \& Butler, R. P. 2002, ApJ, 576, 478

Frink, S., Quirrenbach, A., Fischer, D., Röser, S., \& Schilbach, E. 2001, PASP, 113,173

Gaudi, B. S., Seager, S., \& Mallen-Ornelas, G. 2005, ApJ, 623, 472 (GSM05) Gould, A. 2001, ApJ, 559, 484

Gray, D. F. 1992, The Observation and Analysis of Stellar Photospheres (Cambridge: Cambridge Univ. Press), 370

Gu, P.-G., Lin, D. N. C., \& Bodenheimer, P. H. 2003, ApJ, 588, 509

Henry, G. W. 1999, PASP, 111, 845

Henry, G. W., Baliunas, S. L., Donahue, R. A., Fekel, F. C., \& Soon, W. 2000a, ApJ, 531, 415 
Henry, G. W., Fekel, F. C., \& Hall, D. S. 1995, AJ, 110, 2926

Henry, G. W., Marcy, G. W., Butler, R. P., \& Vogt, S. S. 2000b, ApJ, 529, L41

Ida, S., \& Lin, D. N. C. 2004, ApJ, 604, 388

Jansson, P. 1995, Deconvolution: With Applications in Spectroscopy (New York: Academic Press)

Konacki, M., Torres, G., Jha, S., \& Sasselov, D. D. 2003, Nature, 421, 507

Marcy, G. W., Butler, R. P., Fischer, D., Vogt, S., Wright, J. T., Tinney, C. G., \& Jones, H. R. A. 2005a, Prog. Theor. Phys. Suppl., 158, 24

Marcy, G. W., Butler, R. P., Vogt, S. S., Fischer, D. A., Henry, G. W., Laughlin, G., Wright, J. T., \& Johnson, J. A. 2005b, ApJ, 619, 570

Murray, N., Hansen, B., Holman, M., \& Tremaine, S. 1998, Science, 279, 69

Noyes, R. W., Hartmann, L. W., Baliunas, S. L., Duncan, D. K., \& Vaughan, A. H. 1984, ApJ, 279, 763

Paulson, D. B., Saar, S. H., Cochran, W. D., \& Henry, G. W. 2004, AJ, 127, 1644

Queloz, D., et al. 2001, A\&A, 379, 279

Rasio, F. A., \& Ford, E. B. 1996, Science, 274, 954

Robinson, S. E., Strader, J., Ammons, S. M., Laughlin, G., \& Fischer, D. 2006, ApJ, 637, 1102

Sato, B., Kambe, E., Takeda, Y., Izumiura, H., Masuda, S., \& Ando, H. 2005a, PASJ, 57, 97
Sato, B., et al. 2002, PASJ, 54, 873 2005b, ApJ, 633, 465

Seagroves, S., Harker, J., Laughlin, G., Lacy, J., \& Castellano, T. 2003, PASP, 115,1355

Starck, J. L., Pantin, E., \& Murtagh, F. 2002, PASP, 114, 1051

Trilling, D. E., Benz, W., Guillot, T., Lunine, J. I., Hubbard, W. B., \& Burrows, A. $1998, \mathrm{ApJ}, 500,428$

Udalski, A., et al. 2002, Acta Astron., 52, 1

Udry, S., et al. 2003, A\&A, 407, 679

Valenti, J. A., \& Fischer, D. A. 2005, ApJS, 159, 141

Vogt, S. S., Marcy, G. W., Butler, R. P., \& Apps, K. 2000, ApJ, 536, 902

Vogt, S. S., et al. 1994, Proc. SPIE, 2198, 362

Weidenschilling, S. J., \& Marzari, F. 1996, Nature, 384, 619

Winn, J. N., et al. 2005, ApJ, 631, 1215

Wright, J. T. 2005, PASP, 117, 657

Yi, S., Demarque, P., Kim, Y.-C., Lee, Y.-W., Ree, C. H., Lejeune, T., \& Barnes, S. 2001, ApJS, 136, 417 